\title{
Baseline Tumor Size as a Predictive and Prognostic Factor of Immune Checkpoint Inhibitor Therapy for Non-small Cell Lung Cancer
}

\author{
MASAHIRO KATSURADA, TATSUYA NAGANO, MOTOKO TACHIHARA, TATSUNORI KIRIU, \\ KOICHI FURUKAWA, KIYOKO KOYAMA, TAKEHIRO OTOSHI, REINA SEKIYA, \\ DAISUKE HAZAMA, DAISUKE TAMURA, KYOSUKE NAKATA, NAOKO KATSURADA, \\ MASATSUGU YAMAMOTO, KAZUYUKI KOBAYASHI and YOSHIHIRO NISHIMURA \\ Division of Respiratory Medicine, Department of Internal Medicine, \\ Kobe University Graduate School of Medicine, Kobe, Japan
}

\begin{abstract}
Background/Aim: Immune checkpoint inhibitors (ICI) are a novel medication for non-small cell lung cancer (NSCLC). Recent reports indicated that baseline tumor size (BTS) relates to the efficacy of ICI therapy for melanoma, but no study exists for NSCLC. This study aimed to evaluate the utility of BTS for ICI therapy. Patients and Methods: Data from 58 patients diagnosed with NSCLC who underwent ICI monotherapy, were retrospectively analyzed. Patients were divided into two groups according to BTS (below $101 \mathrm{~mm}$, above $101 \mathrm{~mm})$. The primary endpoint was progression-free survival (PFS) and the secondary endpoint was overall survival (OS). Results: PFS of patients with a large BTS was significantly shorter than that of those with a small BTS (median; 2.07 [95\% confidence interval [CI] =0.99-6.77] months versus 6.39 [95\%CI=4.17-11.50] months) ( $p=0.044)$. OS of patients with large BTS was also significantly shorter $(p<0.01)$. Conclusion: BTS is a predictive and prognostic negative factor of ICI therapy for NSCLC.
\end{abstract}

Lung cancer is one of the leading causes of cancer-related death worldwide (1). Advanced non-small cell lung cancer (NSCLC) is treated using systemic therapies, including chemotherapy, epidermal growth factor receptor-tyrosine

This article is freely accessible online.

Correspondence to: Tatsuya Nagano, MD, Ph.D., Project Assistant Professor of Division of Respiratory Medicine, Department of Internal Medicine, Kobe University Graduate School of Medicine, $7-$ 5-1 Kusunoki-cho, Chuo-ku, Kobe, 650-0017, Japan. Tel: +81 783825660, Fax: +81 783825661, e-mail: tnagano@med.kobe-u.ac.jp

Key Words: Baseline tumor size, tumor burden, non-small cell lung cancer, immune checkpoint inhibitor, progression-free survival, overall survival. kinase inhibitor (TKI), anaplastic lymphoma kinase-TKI, c-ros oncogene 1 inhibitor, a combination of BRAF/MET inhibitor, and an immune checkpoint inhibitor (ICI) (2). The immune system protects the host against cancer cells via seven steps termed the cancer-immunity cycle (3). The seven steps include 'priming and activation' and 'recognition of cancer by T cells' (3). These processes are regulated and activated by various molecules and cytokines, including programmed death-ligand 1 (PD-L1) and programmed death-1 (PD-1), which suppress the cancer-immune cycle $(3,4)$. Cancer cells can express PDL1 on their surfaces, facilitating recognition by $\mathrm{PD}-1$, which is expressed on the surface of T-cells and acts as a PD-L1 receptor. Binding of PD-L1 to T cells has a profound inhibitory effect on immune functions such as cytokine secretion, growth, and cytotoxicity. Hence, PD-1 and PD-L1 are thought to be important target molecules for the control of cancer.

ICI is a novel and promising medication for NSCLC. Nivolumab, a fully human IgG4 anti-PD-1 receptor-blocking monoclonal antibody (5), was the first ICI available for treatment of NSCLC in Japan. The Checkmate 017 and Checkmate 057 studies indicated the superiority of nivolumab over docetaxel for patient progression-free survival (PFS) and overall survival (OS) $(6,7)$. In addition to nivolumab, various other ICIs are now available, including pembrolizumab (an anti-PD-1 blocking antibody), atezolizumab and durvalumab (anti-PD-L1 blocking antibodies). Use of these ICIs has also been shown to lead to superior PFS and OS (8-10).

Previous studies (Checkmate 017, 057, and Keynote-010) showed that the effects of ICI differed according to PD-L1 expression. As ICI is more effective against NSCLC expressing a higher tumor proportion score (TPS) of PD-L1 $(6,7)$, TPS of PD-L1 is considered one of the most useful prognostic biomarkers. However, a recent study indicated that the efficacy of ICI did not necessarily depend on PD-L1 expression (9). Hence, investigators seek more reliable 
predictive and prognostic factors. To date, various factors have been proposed as potential prognostic indicators, including tumor mutation burden (11), presence of liver metastasis (12), gut microbiome (13), and neutrophil-tolymphocyte ratio (14).

Baseline tumor size (BTS), quantified as the sum of the longest dimensions of all measurable target lesions, reflects tumor burden, load, and bulk. In patients undergoing ICI therapy for melanoma, BTS below $102 \mathrm{~mm}$ is associated with significantly better predicted OS (15) and is expected as a new prognostic factor. However, in patients with NSCLC, no study has examined the relationship between BTS, PFS and OS. We hypothesized that BTS is a potential predictive and prognostic indicator also in patients undergoing ICI therapy for NSCLC. The aim of this study was to evaluate the utility of BTS as a simple and useful prognostic factor of ICI treatment in NSCLC patients.

\section{Patients and Methods}

Patients and treatment. A retrospective review of 59 consecutive patients who were diagnosed with NSCLC histologically and treated with ICI monotherapy at Kobe University Hospital in Japan from December 2015 to April 2018 was Performed. Collected data included sex, age, Brinkman index, Eastern Cooperative Oncology Group Performance Status (ECOG PS), BTS, metastatic site, and yStage at the time of initiating ICI, driver mutation, history of surgery, history of radiation therapy, histology, TPS of PD-L1, lines of prior therapy, and types of medication. yStage was based on the eighth edition of the Lung Cancer Stage Classification from the Union for International Cancer Control and the American Joint Committee on Cancer (16). TPS of PD-L1 was assessed using the 22C3 antibody.

To clarify the characteristics of ICI, a retrospective review of consecutive patients who were diagnosed with NSCLC histologically or cytologically and started treatment with chemotherapy from December 2015 to January 2017 was also performed. Patients with driver mutations and who have undergone chemoradiotherapy treatment were excluded. Collected data were same as above.

This retrospective analysis was approved by the Institutional Review Board of Kobe University Hospital (permission number: \#170023).

BTS. BTS was quantified as the sum of the longest dimensions of all measurable target lesions. We summed the longest major axis of all measurable target lesions, except for metastatic lymph nodes, and the longest minor axis of metastatic lymph nodes. Target lesions were basically assessed by Response Evaluation Criteria in Solid Tumors (RECIST) version 1.1, but also unmeasurable target was calculated as far as possible to show BTS more accurately. A maximum of five organs were selected and one or two lesions per organ were measured using a computed tomography scan or magnetic resonance imaging within 1 month of initializing therapy. As mentioned above, in patients with NSCLC, no study exists about the relationship between BTS and PFS. Cutoff points for BTS were tested for PFS using the biostatistical tool, cutoff finder (17). Not only in the case of ICI treatment but also in the case of chemotherapy, the cutoff point of BTS was the same, $101 \mathrm{~mm}$.
Endpoints. The best overall response was assessed according to RECIST version 1.1. The follow-up period was from December 2015 to November 2018. Patients who were still alive and free from progression were censored at the time of last follow-up. The primary endpoint was PFS and the secondary endpoint was OS. Statistical methods. Categorical data are presented as numbers and percentages. Numeric data are presented as median (lower quartile [Q1], upper quartile [Q3]). Univariate analyses were performed using Fisher's exact test for categorical data, and Mann-Whitney U-tests were carried out for analysis of numeric data. PFS and OS curves were estimated using the Kaplan-Meier method and compared using the log-rank test and Cox proportional hazards regression analysis. To investigate prognostic factors for PFS and OS, variables including BTS, ECOG PS, hepatic metastasis, TPS of PD-L1, and lines of prior therapy were analyzed using multivariate Cox proportional hazards regression analysis to test independence in a stepwise procedure with alpha-to-remove, 0.05 . All $p$-values were two-sided and values $<0.05$ were considered statistically significant. All statistical analyses were performed with EZR version 1.37 (Saitama Medical Center Jichi Medical University; http://www.jichi.ac.jp/saitama-sct/SaitamaHP.files/statmed.html; Kanda, 2018), a graphical user interface for R (The R Foundation for Statistical Computing, Vienna, Austria, version 3.4.1) (18).

\section{Results}

Patient characteristics. Patients $(\mathrm{n}=59)$ were treated with ICI monotherapy during the study period. The study included 58 patients, as one patient was excluded because of double primary lung cancers. The baseline characteristics of patients are presented in Table I. The majority of patients (91.4\%) were male. The median age was $70.5(\mathrm{Q} 1, \mathrm{Q} 3: 65.25,74.00)$ years. ECOG PS was 0 or 1 for $84.5 \%$ of patients and $20.7 \%$ of patients received ICI as a primary treatment. The median BTS was 84 (Q1, Q3: 15.10, 208.10) $\mathrm{mm}$. The overall response rate (ORR) of all patients to ICI was $26.3 \%$. Next, patients were divided into two groups according to BTS and their characteristics were retrospectively compared. Patients with large BTS (BTS above $101 \mathrm{~mm}$ ) tended to have hepatic metastasis, poor ECOG PS, and more metastatic sites, but there were no factors with significant differences between the small BTS group and the large BTS group (Table II).

The study included 43 patients treated with chemotherapy. Patients with large BTS tended to have more metastatic sites, there were no factors with significant differences between the small BTS group and the large BTS group (Table III).

Analyses of PFS. The median follow-up time was $9.52(\mathrm{Q} 1$, Q3: 3.29, 14.77) months at the time of analysis. Median PFS was 5.14 $(95 \% \mathrm{CI}=3.32-7.69)$ months at the time of analysis. The median PFS of patients with large BTS was significantly shorter than that of patients with small BTS (2.07 [95\% CI =0.99-6.77] months vs. 6.39 [95\% CI=4.17-11.50] months) ( $p=0.044)$ (Figure 1A). Univariate analysis showed that history of prior chemotherapy, hepatic metastasis, and BTS were significantly associated with PFS (history of prior 
Table I. Characteristics of patients treated with immune checkpoint inhibitors $(n=58)$.

\begin{tabular}{|c|c|}
\hline Factor & Value \\
\hline \multicolumn{2}{|l|}{ Gender } \\
\hline Male & $53(91.4)$ \\
\hline Female & $5(8.6)$ \\
\hline Age $(\text { years })^{\wedge}$ & $70.50(65.25,74.00)$ \\
\hline Brinkman index ${ }^{\wedge}$ & $967.50(712.50,1492.50)$ \\
\hline \multicolumn{2}{|l|}{ ECOG PS } \\
\hline 0 & $1(1.7)$ \\
\hline 1 & $48(82.8)$ \\
\hline 2 & $7(12.1)$ \\
\hline 3 & $1(1.7)$ \\
\hline 4 & $1(1.7)$ \\
\hline \multicolumn{2}{|l|}{ Driver mutation } \\
\hline$E G F R$ & $5(8.6)$ \\
\hline Negative & $52(89.7)$ \\
\hline Unexamined & $1(1.7)$ \\
\hline \multicolumn{2}{|l|}{ History of surgery } \\
\hline Yes & $11(19.0)$ \\
\hline No & $47(81.0)$ \\
\hline \multicolumn{2}{|c|}{ History of radiation therapy } \\
\hline Yes & $15(25.9)$ \\
\hline No & $43(74.1)$ \\
\hline \multicolumn{2}{|l|}{ yStage } \\
\hline IB & $1(1.7)$ \\
\hline IIB & $2(3.4)$ \\
\hline IIIA & $1(1.7)$ \\
\hline IIIB & $6(10.3)$ \\
\hline IIIC & $4(6.9)$ \\
\hline IVA & $13(22.4)$ \\
\hline IVB & $22(37.9)$ \\
\hline Undetermined & $9(15.5)$ \\
\hline \multicolumn{2}{|c|}{ Lines of prior therapy } \\
\hline 0 & $12(20.7)$ \\
\hline 1 & $15(25.9)$ \\
\hline 2 & $17(29.3)$ \\
\hline 3 & $10(17.2)$ \\
\hline 4 & $3(5.2)$ \\
\hline 6 & $1(1.7)$ \\
\hline \multicolumn{2}{|l|}{ Type of histology } \\
\hline Non-Squamous & $40(69.0)$ \\
\hline Squamous & $18(31.0)$ \\
\hline \multicolumn{2}{|c|}{ Tumor Proportion Score of PD-L1 } \\
\hline$<1 \%$ & $8(13.8)$ \\
\hline $1 \%-49 \%$ & $11(19.0)$ \\
\hline$\geq 50 \%$ & $16(27.6)$ \\
\hline Unexamined & $23(39.7)$ \\
\hline \multicolumn{2}{|l|}{ Brain metastases } \\
\hline Yes & $8(13.8)$ \\
\hline No & $42(72.4)$ \\
\hline Unknown & $8(13.8)$ \\
\hline \multicolumn{2}{|l|}{ Hepatic metastases } \\
\hline Yes & $7(12.1)$ \\
\hline No & $43(74.1)$ \\
\hline Unknown & $8(13.8)$ \\
\hline \multicolumn{2}{|l|}{ Bone metastases } \\
\hline Yes & $11(19.0)$ \\
\hline No & $39(67.2)$ \\
\hline Unknown & $8(13.8)$ \\
\hline
\end{tabular}

\begin{tabular}{lc}
\hline Factor & Value \\
\hline No. of metastatic sites & \\
0 & $3(6.1)$ \\
1 & $16(32.7)$ \\
2 & $16(32.7)$ \\
3 & $8(16.3)$ \\
4 & $3(6.1)$ \\
5 & $2(4.1)$ \\
6 & $1(2.0)$ \\
Baseline tumor size^ & $84.00(15.10,208.10)$ \\
Medication type of ICI & \\
Nivolumab & $38(65.5)$ \\
Pembrolizumab & $20(34.5)$ \\
Response & $15(26.3)$ \\
PR & $23(39.7)$ \\
SD & $17(29.8)$ \\
PD & $3(5.2)$ \\
Not evaluated &
\end{tabular}

Values are presented as $\mathrm{n}(\%)$, except those variables marked with the symbol $\wedge^{\wedge}$ which are presented as median (lower quartile, upper quartile). ECOG PS: Eastern Cooperative Oncology Group Performance Status; EGFR: Epidermal growth factor receptor; PD-L1: programmed deathligand 1; ICI: immune checkpoint inhibitor; PR: partial response; SD: stable disease; PD: progressive disease.

chemotherapy, Hazard Ratio (HR) $=2.594[95 \% \mathrm{CI}=1.093-$ 6.152]; hepatic metastasis, $\mathrm{HR}=3.286$ [95\% $\mathrm{CI}=1.412-7.646]$; large BTS, $\mathrm{HR}=1.818$ [95\% CI=1.005-3.291]). Multivariate analysis with Cox hazard regression was performed by incorporating ECOG PS, hepatic metastasis, history of prior chemotherapy, TPS of PD-L1, and BTS and showed that history of prior chemotherapy and BTS were significant independent prognostic factors for PFS (history of prior chemotherapy, HR=3.017 [95\% CI=1.259-7.229]; large BTS, $\mathrm{HR}=2.117$ [95\%CI=1.162-3.859]) (Table IV).

The median PFS of patients between the small BTS group and the large BTS group was not significantly different [4.99 $(95 \% \mathrm{CI}=2.69-5.36)$ months in the small BTS group $v s .5 .52$ (95\% CI $=1.41-9.49)$ months in the large BTS group $(p=0.329)$ ]. Moreover, the Kaplan-Meier (KM) curves between these groups were crossed (Figure 2).

Analyses of OS. Median OS was 14.03 (95\%CI=8.51-22.34) months. The median OS of patients with large BTS was significantly shorter than that of patients with small BTS [5.85 (95\% CI $=2.00-10.68)$ months vs. 22.28 (95\% CI=14.03-not reached) months $(p<0.01)$ ] (Figure 1B). Univariate analysis showed that ECOG PS, hepatic metastasis, and BTS were significantly associated with OS [ECOG PS $\geq 2, \mathrm{HR}=2.699$ $(95 \% \mathrm{CI}=1.074-6.785)$; hepatic metastasis, $\mathrm{HR}=4.503$ $(95 \% \mathrm{CI}=1.700-11.96)$; large BTS, HR=3.122 $(95 \% \mathrm{CI}=1.516-$ 6.426)]. Multivariate analysis with Cox hazard regression was 
Table II. Characteristics of patients treated with immune checkpoint inhibitors according to basement tumor size (BTS).

\begin{tabular}{|c|c|c|c|}
\hline Factor & $\begin{array}{c}\text { Small (below } \\
101 \mathrm{~mm} \text { ) } \\
\mathrm{n}=34\end{array}$ & $\begin{array}{c}\text { Large (above } \\
\begin{array}{c}101 \mathrm{~mm} \text { ) } \\
\mathrm{n}=24\end{array}\end{array}$ & $p$-Value \\
\hline Gender & & & 0.64 \\
\hline Male & $32(94.1)$ & $21(87.5)$ & \\
\hline Female & $2(5.9)$ & $3(12.5)$ & \\
\hline $\mathrm{Age}^{\wedge}$ & $71.0(67.25,75.75)$ & $70.0(63.00,74.00)$ & 0.385 \\
\hline Brinkman index ${ }^{\wedge}$ & $970.0(669.0,1350)$ & $937.5(669.0,1650)$ & 0.782 \\
\hline ECOG PS & & & 0.059 \\
\hline 0 & $1(2.9)$ & $0(0.0)$ & \\
\hline 1 & $31(91.1)$ & $17(70.8)$ & \\
\hline 2 & $2(5.9)$ & $5(20.8)$ & \\
\hline 3 & $0(0.0)$ & $1(4.2)$ & \\
\hline 4 & $0(0.0)$ & $1(4.2)$ & \\
\hline Driver mutation & & & 1 \\
\hline EGFR & $3(8.8)$ & $2(8.3)$ & \\
\hline Negative & $30(88.2)$ & $22(91.7)$ & \\
\hline Unexamined & $1(2.9)$ & $0(0.0)$ & \\
\hline History of surgery & & & 1 \\
\hline Yes & $7(20.6)$ & $4(16.7)$ & \\
\hline No & $27(79.4)$ & $20(83.3)$ & \\
\hline History of & & & \\
\hline radiation therapy & & & 0.552 \\
\hline Yes & $10(29.4)$ & $5(20.8)$ & \\
\hline No & $24(70.6)$ & $19(79.2)$ & \\
\hline yStage & & & 0.462 \\
\hline IB & $1(2.9)$ & $0(0.0)$ & \\
\hline IIB & $2(5.9)$ & $0(0.0)$ & \\
\hline IIIA & $0(0.0)$ & $1(4.2)$ & \\
\hline IIIB & $4(11.8)$ & $2(8.3)$ & \\
\hline IIIC & $2(5.9)$ & $2(8.3)$ & \\
\hline IVA & $10(29.4)$ & $3(12.5)$ & \\
\hline IVB & $11(32.4)$ & $11(45.8)$ & \\
\hline Undetermined & $4(11.8)$ & $5(20.8)$ & \\
\hline Lines of prior therapy & & & 0.156 \\
\hline 0 & $6(17.6)$ & $6(25.0)$ & \\
\hline 1 & $11(32.4)$ & $4(16.7)$ & \\
\hline 2 & $12(35.3)$ & $5(20.8)$ & \\
\hline 3 & $3(8.8)$ & $7(29.2)$ & \\
\hline 4 & $2(5.9)$ & $1(4.2)$ & \\
\hline 6 & $0(0.0)$ & $1(4.2)$ & \\
\hline Type of histology & & & 0.249 \\
\hline Non-squamous & $21(61.8)$ & $19(79.2)$ & \\
\hline Squamous & $13(38.2)$ & $5(20.8)$ & \\
\hline $\begin{array}{l}\text { Tumor proportion } \\
\text { score of PD-L1 }\end{array}$ & & 0.149 & \\
\hline$<1 \%$ & $5(14.7)$ & $3(12.5)$ & \\
\hline $1-49 \%$ & $9(26.4)$ & $2(8.3)$ & \\
\hline$\geq 50 \%$ & $6(17.6)$ & $10(41.7)$ & \\
\hline Unexamined & $14(41.2)$ & $9(37.5)$ & \\
\hline Brain metastases & & & 0.477 \\
\hline Yes & $5(14.7)$ & $3(12.5)$ & \\
\hline No & $26(76.5)$ & $16(66.7)$ & \\
\hline Unknown & $3(8.8)$ & $5(20.8)$ & \\
\hline Hepatic metastases & & & 0.075 \\
\hline Yes & $2(5.9)$ & $5(20.8)$ & \\
\hline No & $29(85.3)$ & $14(58.3)$ & \\
\hline Unknown & $3(8.8)$ & $5(20.8)$ & \\
\hline
\end{tabular}

\begin{tabular}{lccc}
\hline Factor & $\begin{array}{c}\text { Small (below } \\
101 \mathrm{~mm}) \\
\mathrm{n}=34\end{array}$ & $\begin{array}{c}\text { Large (above } \\
101 \mathrm{~mm}) \\
\mathrm{n}=24\end{array}$ & $p$-Value \\
\hline Bone metastases & & & 0.369 \\
Yes & $6(17.6)$ & $5(20.8)$ & \\
No & $25(73.5)$ & $14(58.3)$ & \\
Unknown & $3(8.8)$ & $5(20.8)$ & \\
No. of metastatic sites & & & 0.085 \\
0 & $2(5.9)$ & $1(4.2)$ & \\
1 & $12(35.3)$ & $4(16.7)$ & \\
2 & $12(35.3)$ & $4(16.7)$ & \\
3 & $3(8.8)$ & $5(20.8)$ & \\
4 & $1(3.4)$ & $2(8.3)$ & \\
5 & $0(0.0)$ & $2(8.3)$ & \\
6 & $0(0.0)$ & $1(4.2)$ & 0.165 \\
Type of medication & & & \\
Nivolumab & $25(73.5)$ & $13(54.2)$ & \\
Pembrolizumab & $9(26.4)$ & $11(45.8)$ & \\
Response & & & \\
PR & $11(32.4)$ & $4(16.7)$ & \\
SD & $16(47.1)$ & $7(29.2)$ & \\
PD & $6(17.6)$ & $11(45.8)$ & \\
unknown & $1(3.4)$ & $2(8.3)$ & \\
\hline
\end{tabular}

Values are presented as $\mathrm{n}(\%)$, except those variables marked with the symbol ${ }^{\wedge}$ which are presented as median (lower quartile, upper quartile). $* p<0.05$. ECOG PS: Eastern Cooperative Oncology Group Performance Status; EGFR: epidermal growth factor receptor; PD-L1: programmed death-ligand 1; PR: partial response; SD: stable disease; PD: progressive disease.

performed by incorporating ECOG PS, hepatic metastasis, history of prior chemotherapy, TPS of PD-L1, and BTS and showed that ECOG PS $\geq 2$, history of prior chemotherapy, and BTS were significant independent prognostic factors for OS [ECOG PS $\geq 2, \mathrm{HR}=3.407$ (95\%CI=1.180-9.841); history of prior chemotherapy, $\mathrm{HR}=4.044(95 \% \mathrm{CI}=1.273-12.85)$; large BTS, HR=3.090 $(95 \% \mathrm{CI}=1.456-6.558)]($ Table V).

Response of ICI therapy and treatment after ICI therapy. Next, to investigate the reason why the OS of patients with small BTS was significantly longer than that of patients with large BTS, we analyzed the differences in the response of ICI therapy and posttreatment between the small BTS group and the large BTS group. The ORR of ICI therapy was not significantly different between the small BTS group and the large BTS group (32.4\% in the small BTS group vs. $16.7 \%$ in the large BTS group) $(p=0.231)$, however the proportion of PD of ICI therapy was significantly larger in the large BTS group (17.6\% in the small BTS group $v s .45 .8 \%$ in the large BTS group) $(p=0.039)$. At the time of analysis, the majority of patients with large BTS received best supportive care after getting PD of ICI therapy $(p<0.01)$. The response of post chemotherapy was not significantly different between these groups $(p=0.727)$ (Table VI). 
Table III. Characteristics of patients treated with chemoterapy according to basement tumor size (BTS).

\begin{tabular}{|c|c|c|c|}
\hline Factor & $\begin{array}{c}\text { Small (below } \\
101 \mathrm{~mm} \text { ) } \\
n=30\end{array}$ & $\begin{array}{c}\text { Large (above } \\
101 \mathrm{~mm}) \\
\mathrm{n}=13\end{array}$ & $p$-Value \\
\hline Gender & & & 0.649 \\
\hline Male & $25(83.3)$ & $12(92.3)$ & \\
\hline Female & $5(16.7)$ & $1(7.7)$ & \\
\hline $\mathrm{Age}^{\wedge}$ & $69.5(63.5,73.8)$ & $68.0(66.0,70.0)$ & 0.791 \\
\hline Brinkman index ${ }^{\wedge}$ & $840.0(600.0,1015)$ & $990.0(810.0,1040)$ & 0.442 \\
\hline ECOG PS & & & 0.095 \\
\hline 0 & $8(26.7)$ & $0(0.0)$ & \\
\hline 1 & $17(56.7)$ & $9(69.2)$ & \\
\hline 2 & $5(16.7)$ & $4(30.8)$ & \\
\hline History of surgery & & & 0.681 \\
\hline Yes & $5(16.7)$ & $3(23.1)$ & \\
\hline No & $25(83.3)$ & $10(76.9)$ & \\
\hline History of radiation & & & \\
\hline $\begin{array}{l}\text { therapy } \\
\text { Yes }\end{array}$ & $12(40.0)$ & $2(15.4)$ & 0.164 \\
\hline No & $18(60.0)$ & $11(84.6)$ & \\
\hline yStage & & & 0.235 \\
\hline IB & $3(10.0)$ & $0(0.0)$ & \\
\hline IIB & $1(3.3)$ & $0(0.0)$ & \\
\hline IIIA & $2(6.7)$ & $0(0.0)$ & \\
\hline IIIB & $0(0.0)$ & $2(15.4)$ & \\
\hline IIIC & $3(10.0)$ & $1(7.7)$ & \\
\hline IVA & $9(30.0)$ & $2(15.4)$ & \\
\hline IVB & $12(40.0)$ & $8(61.5)$ & \\
\hline Type of histology & & & 0.696 \\
\hline Non-squamous & $22(73.3)$ & $11(84.6)$ & \\
\hline Squamous & $8(26.7)$ & $2(15.4)$ & \\
\hline \multicolumn{4}{|l|}{ Tumor proportion } \\
\hline score of PD-L1 & & 1 & \\
\hline$<1 \%$ & $2(6.7)$ & $1(7.7)$ & \\
\hline $1-49 \%$ & $3(10.0)$ & $0(0.0)$ & \\
\hline$\geq 50 \%$ & $0(0.0)$ & $0(0.0)$ & \\
\hline Unexamined & $25(83.3)$ & $12(92.3)$ & \\
\hline
\end{tabular}

\section{Discussion}

This study suggests that BTS is a useful negative predictive and prognostic marker not only for PFS but also for OS of NSCLC patients treated with nivolumab or pembrolizumab monotherapy. Among chemotherapy patients, KM curves between the small and the large BTS groups were crossed, suggesting that BTS is a specific marker of ICI monotherapy. To our knowledge, this is the first report of an association between BTS and ICI treatment in NSCLC.

For patients with melanoma, Joseph et al. showed that patients with small BTS had significantly better OS compared to those with large BTS (15). Similarly, we showed that patients with NSCLC and a large BTS had significantly inferior PFS and OS.

\begin{tabular}{lccc}
\hline Factor & $\begin{array}{c}\text { Small (below } \\
101 \mathrm{~mm}) \\
\mathrm{n}=30\end{array}$ & $\begin{array}{c}\text { Large (above } \\
101 \mathrm{~mm}) \\
\mathrm{n}=13\end{array}$ & $p$-Value \\
& & & \\
\hline Brain metastases & $5(16.7)$ & $1(7.7)$ & 0.649 \\
Yes & $25(83.3)$ & $12(92.3)$ & \\
No & & & 0.172 \\
Hepatic metastases & $3(10.0)$ & $4(30.8)$ & \\
Yes & $27(90.0)$ & $9(69.2)$ & \\
No & & & 0.105 \\
Bone metastases & $10(33.3)$ & $8(61.5)$ & \\
Yes & $20(66.7)$ & $5(38.5)$ & \\
No & & & 0.097 \\
No. of metastatic sites & $4(13.3)$ & $0(0.0)$ & \\
0 & $8(26.7)$ & $3(23.1)$ & \\
1 & $12(40.0)$ & $2(15.4)$ & \\
2 & $3(10.0)$ & $3(23.1)$ & \\
3 & $2(6.7)$ & $4(30.8)$ & \\
4 & $1(3.3)$ & $1(7.7)$ & \\
5 & $0(0.0)$ & $0(0.0)$ & \\
6 & & & \\
Type of medication & $22(73.3)$ & $7(53.8)$ & \\
Platinum doublet & $8(26.7)$ & $6(46.2)$ & \\
Non-platinum doublet & & & \\
Response & & $2(15.4)$ & \\
PR & $10(33.3)$ & $4(30.8)$ & \\
SD & $12(40.0)$ & $3(30.8)$ & \\
PD & $8(26.7)$ & & \\
Unknown & $0(0.0)$ & & \\
\hline
\end{tabular}

Values are presented as $\mathrm{n}(\%)$, except those variables marked with the symbol $\wedge^{\wedge}$ which are presented as median (lower quartile, upper quartile). ECOG PS: Eastern Cooperative Oncology Group Performance Status; PD-L1: programmed death-ligand 1; PR: partial response; SD: stable disease; PD: progressive disease.

This study examined why PFS and OS in the large BTS group is worse than the small BTS group. Table V shows that, compared to the small BTS group, in the large BTS group there was a higher proportion of PD and less patients continued ICI therapy. This may be partly attributed to the fact that BTS reflects tumor burden (i.e., tumor bulk) and high tumor burden is associated with an inferior prognosis and effect of ICI. In contrast, in the current study yStage was not found to be a significant marker for PFS nor OS. These results suggest that BTS contributes to survival because of its relationship with tumor burden, rather than with yStage. Kobayashi $\mathrm{H}$ et al. reported that the responders to prior chemotherapy with nivolumab had benefited from ICI (19). Although there are no actual data, it is suggested that the tumor burden of responders to prior chemotherapy is less than that of non-responders.

The predictive and prognostic value of BTS for PFS and OS may be due to first, tumor associated antigen (TAA) retention, second, the immunosuppressive effect of the tumor, and third, an imbalance between the immune response and the tumor. 
Table IV. Progression-free survival of patients undergoing immune checkpoint inhibitor therapy.

\begin{tabular}{|c|c|c|c|c|}
\hline Univariate analysis & $\mathrm{n}$ & 1-year survival rate $(\%)(95 \% \mathrm{CI})$ & Hazard ratio $(95 \% \mathrm{CI})$ & $p$-Value \\
\hline Gender & & & & 0.222 \\
\hline Female & 5 & $20.0(0.8-58.2)$ & 1 & \\
\hline Male & 53 & $24.9(14.0-37.4)$ & $0.532(0.189-1.495)$ & \\
\hline Brinkman index & & & & 0.933 \\
\hline Below median & 29 & $23.7(9.5-41.5)$ & 1 & \\
\hline Above median & 29 & $24.1(10.7-40.5)$ & $0.975(0.542-1.755)$ & \\
\hline ECOG PS & & & & 0.366 \\
\hline 0,1 & 49 & $24.8(13.5-37)$. & 1 & \\
\hline $2,3,4$ & 9 & $22.2(3.4-51.3)$ & $1.450(0.643-3.268)$ & \\
\hline History of surgery & & & & 0.947 \\
\hline Yes & 11 & $27.3(6.5-53.9)$ & 1 & \\
\hline No & 47 & $23.6(12.3-36.9)$ & $0.974(0.451-2.105)$ & \\
\hline History of radiation therapy & & & & 0.074 \\
\hline Yes & 15 & $6.7(0.4-26.0)$ & 1 & \\
\hline No & 43 & $31.0(17.7-45.4)$ & $0.562(0.295-1.069)$ & \\
\hline yStage & & & & 0.203 \\
\hline IB-IIIC & 14 & $50.0(22.9-72.2)$ & 1 & \\
\hline IVA, IVB & 35 & $14.2(4.8-28.7)$ & $2.007(0.912-4.415)$ & \\
\hline Undetermined & 9 & $22.2(3.4-51.3)$ & $1.559(0.561-4.337)$ & \\
\hline History of prior chemotherapy & & & & $0.024 *$ \\
\hline No & 12 & $45.0(15.5-71.0)$ & 1 & \\
\hline Yes & 46 & $19.0(9.1-31.5)$ & $2.594(1.093-6.152)$ & \\
\hline Type of histology & & & & 0.462 \\
\hline Non-squamous & 40 & $24.1(12.2-38.3)$ & 1 & \\
\hline Squamous & 18 & $24.3(7.1-46.9)$ & $0.789(0.419-1.488)$ & \\
\hline Tumor proportion score of PD-L1 & & & & 0.208 \\
\hline$\geq 50 \%$ & 16 & $41.0(16.8-64.1)$ & $0.363(0.139-0.950)$ & \\
\hline $1 \%-49 \%$ & 11 & $27.3(6.5-53.9)$ & $0.580(0.217-1.551)$ & \\
\hline$<1 \%$ & 8 & not available & 1 & \\
\hline Unexamined & 23 & $21.7(7.9-39.9)$ & $0.622(0.270-1.432)$ & \\
\hline Brain metastasis & & & & 0.718 \\
\hline Yes & 8 & $18.8(1.1-53.5)$ & $1.325(0.582-3.015)$ & \\
\hline No & 42 & $25.4(13.3-39.4)$ & 1 & \\
\hline Unknown & 8 & $25.0(3.7-55.8)$ & $0.862(0.359-2.068)$ & \\
\hline Hepatic metastasis & & & & $0.011 *$ \\
\hline Yes & 7 & not available & $3.286(1.412-7.646)$ & \\
\hline No & 43 & $29.9(16.1-43.1)$ & 1 & \\
\hline Unknown & 8 & $25.0(3.7-55.8)$ & $0.945(0.393-2.272)$ & \\
\hline Bone metastasis & & & & 0.865 \\
\hline Yes & 11 & $9.1(0.5-33.3)$ & $1.180(0.544-2.300)$ & \\
\hline No & 39 & $30.3(16.7-45.0)$ & 1 & \\
\hline Unknown & 8 & $25.0(3.7-55.8)$ & $0.878(0.350-2.051)$ & \\
\hline No. of metastatic sites & & & & 0.714 \\
\hline$\leq 2$ & 35 & $31.4(17.1-46.8)$ & $0.756(0.381-1.503)$ & \\
\hline$\geq 3$ & 14 & $8.6(0.5-31.5)$ & 1 & \\
\hline Unknown & 9 & $22.2(3.4-51.3)$ & $0.770(0.302-1.960)$ & \\
\hline Baseline tumor size & & & & $0.044 *$ \\
\hline Small (below $101 \mathrm{~mm}$ ) & 34 & $31.3(16.5-47.3)$ & 1 & \\
\hline Large (above $101 \mathrm{~mm}$ ) & 24 & $14.6(3.8-32.1)$ & $1.818(1.005-3.291)$ & \\
\hline Type of medication & & & & 0.097 \\
\hline Nivolumab & 38 & $17.5(7.4-31.3)$ & 1 & \\
\hline Pembrolizumab & 20 & $38.1(17.2-58.9)$ & $0.575(0.296-1.117)$ & \\
\hline Multivariate analysis & & Hazard ratio & $95 \% \mathrm{CI}$ & $p$-Value \\
\hline Large baseline tumor size & & 2.117 & $1.162-3.859$ & $0.014 *$ \\
\hline History of prior chemotherapy & & 3.017 & $1.259-7.229$ & $0.013 *$ \\
\hline
\end{tabular}

Multivariate analysis included the factors ECOG PS, hepatic metastases, baseline tumor size, history of prior chemotherapy, tumor proportion score. CI: Confidence interval; ECOG PS: Eastern Cooperative Oncology Group Performance Status; PD-L1: programmed death-ligand $1 .{ }^{*} p<0.05,{ }^{\dagger} p<0.01$. 
Table V. Overall survival after immune checkpoint inhibitor therapy.

\begin{tabular}{|c|c|c|c|c|}
\hline \multirow{2}{*}{$\begin{array}{l}\text { Factor } \\
\text { Univariate analysis }\end{array}$} & \multirow[b]{2}{*}{$\mathrm{n}$} & \multicolumn{3}{|c|}{ Overall survival } \\
\hline & & 1-year survival rate $(\%)(95 \% \mathrm{CI})$ & Hazard ratio $(95 \% \mathrm{CI})$ & $p$-Value \\
\hline Gender & & & & 0.413 \\
\hline Female & 5 & $40.0(5.2-75.3)$ & 1 & \\
\hline Male & 53 & $51.7(36.2-65.1)$ & $0.609(0.183-2.026)$ & \\
\hline Brinkman index & & & & 0.808 \\
\hline Below median & 29 & $49.6(28.6-67.5)$ & 1 & \\
\hline Above median & 29 & $52.8(32.6-69.5)$ & $0.916(0.450-1.863)$ & \\
\hline ECOG PS & & & & $0.028 *$ \\
\hline 0,1 & 49 & $54.6(38.4-68.2)$ & 1 & \\
\hline $2,3,4$ & 9 & $29.6(5.2-60.7)$ & $2.699(1.074-6.785)$ & \\
\hline History of surgery & & & & 0.916 \\
\hline Yes & 11 & $42.4(13.7-69.1)$ & 1 & \\
\hline No & 47 & $52.7(36.1-66.8)$ & $1.107(0.415-2.952)$ & \\
\hline History of radiation therapy & & & & 0.408 \\
\hline Yes & 15 & $41.6(16.1-65.6)$ & 1 & \\
\hline No & 43 & $54.8(37.8-69.0)$ & $0.728(0.342-1.551)$ & \\
\hline yStage & & & & 0.724 \\
\hline IB-IIIC & 14 & $64.3(34.3-83.3)$ & 1 & \\
\hline IVA, IVB & 35 & $44.0(25.3-61.3)$ & $1.329(0.559-3.162)$ & \\
\hline Undetermined & 9 & $53.3(17.7-79.6)$ & $0.954(0.279-3.271)$ & \\
\hline History of prior chemotherapy & & & & 0.116 \\
\hline No & 12 & $72.9(36.8-90.5)$ & 1 & \\
\hline Yes & 46 & $44.9(29.0-59.6)$ & $2.276(0.793-6.531)$ & \\
\hline Type of histology & & & & 0.384 \\
\hline Non-squamous & 40 & $53.6(35.8-68.5)$ & 1 & \\
\hline Squamous & 18 & $45.8(20.9-67.8)$ & $1.371(0.671-2.805)$ & \\
\hline Tumor proportion score of PD-L1 & & & & 0.801 \\
\hline$\geq 50 \%$ & 16 & $54.7(27.4-75.5)$ & $0.833(0.242-2.862)$ & \\
\hline $1 \%-49 \%$ & 11 & $63.6(29.7-84.5)$ & $0.667(0.167-2.671)$ & \\
\hline$<1 \%$ & 8 & $56.2(14.7-84.2)$ & 1 & \\
\hline Unexamined & 23 & $43.7(22.6-63.2)$ & $1.117(0.364-3.428)$ & \\
\hline Brain metastases & & & & 0.771 \\
\hline Yes & 8 & $75.0(31.5-93.1)$ & $0.688(0.233-2.030)$ & \\
\hline No & 42 & $46.8(29.8-62.1)$ & 1 & \\
\hline Unknown & 8 & $50.0(31.5-93.1)$ & $0.837(0.288-2.433)$ & \\
\hline Hepatic metastases & & & & $<0.01 \dagger$ \\
\hline Yes & 7 & not available & $4.503(1.700-11.96)$ & \\
\hline No & 43 & $59.2(41.9-73.0)$ & 1 & \\
\hline Unknown & 8 & $50.0(15.2-77.5)$ & $1.085(0.369-3.198)$ & \\
\hline Bone metastases & & & & 0.874 \\
\hline Yes & 11 & $38.4(10.3-66.8)$ & $1.228(0.516-2.920)$ & \\
\hline No & 39 & $54.8(36.5-69.9)$ & 1 & \\
\hline Unknown & 8 & $50.0(15.2-77.5)$ & $0.929(0.316-2.733)$ & \\
\hline No. of metastatic sites & & & & 0.878 \\
\hline$\leq 2$ & 35 & $54.4(35.7-69.8)$ & 1 & \\
\hline$\geq 3$ & 14 & $41.9(14.3-67.8)$ & $0.911(0.393-2.111)$ & \\
\hline Unknown & 9 & $53.3(17.7-79.6)$ & $0.729(0.215-2.472)$ & \\
\hline Baseline tumor size & & & & $<0.01 \dagger$ \\
\hline Small (below 101 mm) & 34 & $71.0(51.2-83.9)$ & 1 & \\
\hline Large (above $101 \mathrm{~mm}$ ) & 24 & $23.0(7.8-42.7)$ & $3.122(1.516-6.426)$ & \\
\hline Type of medication & & & & 0.212 \\
\hline Nivolumab & 38 & $44.6(27.4-60.5)$ & 1 & \\
\hline Pembrolizumab & 20 & $62.2(35.7-80.4)$ & $0.586(0.250-1.373)$ & \\
\hline Multivariate analysis & & Hazard ratio & $95 \% \mathrm{CI}$ & $p$-Value \\
\hline ECOG.PS 2,3,4 & & 3.407 & $1.180-9.841$ & $0.024 *$ \\
\hline Large baseline tumor size & & 3.09 & $1.456-6.558$ & $<0.01^{\dagger}$ \\
\hline History of prior chemotherapy & & 4.044 & $1.273-12.85$ & $0.017 *$ \\
\hline
\end{tabular}

Multivariate analysis included the factors ECOG PS, hepatic metastases, baseline tumor size, history of prior chemotherapy, and tumor proportion score. CI: Confidence interval; ECOG PS: Eastern Cooperative Oncology Group Performance Status; PD-L1: programmed death-ligand 1. ${ }^{*} p<0.05$; ${ }^{\dagger} p<0.01$. 
Table VI. Response of immune checkpoint inhibitor therapy and post treatment.

\begin{tabular}{lccc}
\hline & \multicolumn{3}{c}{ Baseline tumor size } \\
\cline { 2 - 4 } Factor & $\begin{array}{c}\text { Small (below } \\
101 \mathrm{~mm}) \\
\mathrm{n}=34\end{array}$ & $\begin{array}{c}\text { Large (above } \\
101 \mathrm{~mm}) \\
\mathrm{n}=24\end{array}$ & $p$-Value \\
& & & $0.039^{*}$ \\
\hline Response of ICI & $28(82.4)$ & $13(54.2)$ & \\
PR/SD/NE & $6(17.6)$ & $11(45.8)$ & \\
PD & & $0.019^{*}$ & \\
Post treatment after ICI therapy & $10(29.4)$ & $2(8.3)$ & \\
Chemotherapy & $13(38.2)$ & $18(75.0)$ \\
Best supportive care & $11(32.4)$ & $4(16.7)$ \\
Ongoing ICI therapy & $\mathrm{n}=10$ & $\mathrm{n}=2$ & 0.727 \\
Response of post chemotherapy & $3(30.0)$ & $0(0.0)$ \\
PR & $1(10.0)$ & $0(0.0)$ \\
SD & $4(40.0)$ & $2(100.0)$ \\
PD & $2(20.0)$ & $0(0.0)$ & \\
NE &
\end{tabular}

Values are presented as $\mathrm{n}(\%) .{ }^{*} p<0.05,{ }^{\dagger} p<0.01$. ICI: Immune checkpoint inhibitor; PR: partial response; SD: stable disease; PD: progressive disease; NE: not examined.

Interstitial fluid pressure (IFP) within tumors increases with cancer progression. Raju et al. (20) measured lymphatic vessel area using an antibody against lymphatic vessel endothelial hyaluronic acid receptor (LYVE)-1, a lymphatic system specific marker, in rat tongue cancer. Their results demonstrated that the highest IFP values were measured in rats with large tumors and that lymphatic vessel area, determined by LYVE-1 antibody staining, was significantly increased in the peritumoral, compared with the intratumoral, area (20). In addition, when IFP in the tumor is high, intratumoral lymphatic vessels are prone to collapse (21). Consequently, it becomes difficult for antigen-presenting cells recognizing TAAs to migrate into the lymphatic system and remain in the tumor tissue.

Tumors actively suppress the immune response as they grow, using various mechanisms other than the PD-1/PD-L1 pathway. As tumors grow, IFP elevates and the tumor immune microenvironment (TIME) becomes hypoxic with a low $\mathrm{pH}$. Tumor hypoxia leads to expression of hypoxia-inducible factor1 (HIF-1), which induces vascular endothelial growth factor-A (VEGF-A) (22). VEGF-A promotes the expression of PD-1, Tcell immunoglobulin and mucin-domain containing-3 (TIM-3), and cytotoxic T-lymphocyte-associated protein 4 (CTLA-4) on cytotoxic $\mathrm{T}$ lymphocytes (CTLs), leading to a more immunosuppressive TIME $(21,23)$. VEGF-A also inhibits dendritic cell maturation and antigen presentation (21). Therefore, high HIF-1 expression levels are associated with poor prognosis for patients with NSCLC (24). Expanding tumors also produce numerous cytokines, including $\mathrm{CC}$ chemokine ligand (CCL)2, CCL5, CCL21, and CCL28. These chemokines induce tumor progression; for example, CCL2 and CCL5 promote tumor cell proliferation and metastasis, respectively (25), while CCL21 and CCL28 attract naïve and regulatory $\mathrm{T}$ cells to the $\operatorname{TIME}(23,26)$. Tumors also produce granulocyte colony stimulating factor, which is reported to increase the immunosuppressive function of $\mathrm{Gr}-1^{+} \mathrm{CK} 11 \mathrm{~b}^{+}$ myeloid cells (27). Low $\mathrm{pH}$ in the TIME induces regulatory $\mathrm{T}$ cells and myeloid-derived suppressor cells, while inhibiting natural killer cells and CTLs (28), thereby inducing immunosuppression. These facts demonstrate that a higher tumor burden can induce an immunosuppressive response.

As tumors progress, the immune-cancer balance is shifted. Huang et al. (29) found that levels of $\mathrm{Ki67} 7^{+} \mathrm{CD} 8 \mathrm{~T}$ cells increase after administration of pembrolizumab and that these cells were strongly correlated with tumor burden, with an even greater correlation observed following pembrolizumab treatment. The authors proposed that $\mathrm{Ki} 7^{+}$ is a good prognostic marker for OS of melanoma patients treated with pembrolizumab; however, Ki67 ${ }^{+}$expression $>6.5 \%$ was a poor prognostic marker for PFS and OS, while a Ki67/tumor burden ratio $>1.94$ was a good prognostic marker (29). These results suggest that $\mathrm{T}$ cell surface markers are not useful prognostic markers for patients with high tumor burden and that tumor burden is a more important marker than cell surface molecules.

It should be noted that this study was retrospective and conducted at a single-institution; however, however, our results should be reliable because they reproduce the observations reported for patients with melanoma. In addition, BTS is also likely to be a useful and practical negative marker because clinicians routinely calculate the sum of the diameters of tumors when evaluating RECIST. Furthermore, measurement of BTS does not require a specialist or expensive equipment, as needed to perform next-generation sequencing and immunostaining assays.

In conclusion, BTS is a useful and practical predictive and negative prognostic factor during ICI therapy for NSCLC.

\section{Conflicts of Interest}

None of the Authors have any conflict of interest to declare regarding this study.

\section{References}

1 Torre LA, Bray F, Siegel RL, Ferlay J, Lortet-Tieulent J and Jemal A: Global cancer statistics, 2012. CA Cancer J Clin 65(2): 87-108, 2015. PMID: 25651787, DOI: 10.3322/caac.21262

2 Hanna N, Johnson D, Temin S, Baker S Jr., Brahmer J, Ellis PM, Giaccone G, Hesketh PJ, Jaiyesimi I, Leighl NB, Riely GJ, Schiller JH, Schneider BJ, Smith TJ, Tashbar J, Biermann WA and Masters G: Systemic therapy for stage iv non-small-cell lung cancer: American society of clinical oncology clinical practice guideline update. J Clin Oncol 35(30): 3484-3515, 2017. PMID: 28806116, DOI: 10.1200/JCO.2017.74.6065 


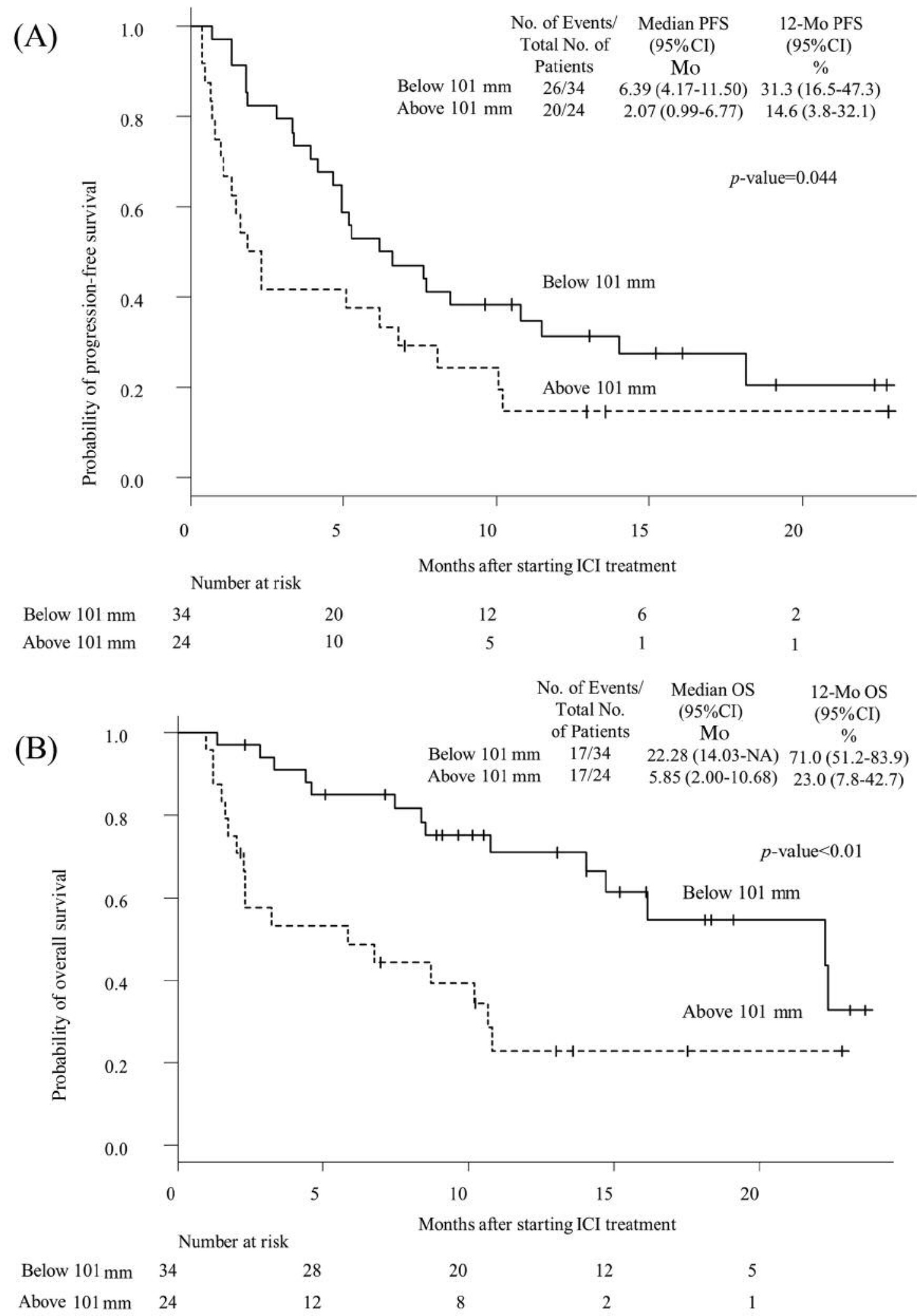

Figure 1. Kaplan-Meier (KM) plots grouped by patients with below and above median Baseline Tumor Size. (A) KM plots for progression-free survival (PFS) after starting immune checkpoint inhibitor therapy. (B) KM plots for overall survival (OS) after starting immune checkpoint inhibitor therapy. Mo: Months. 


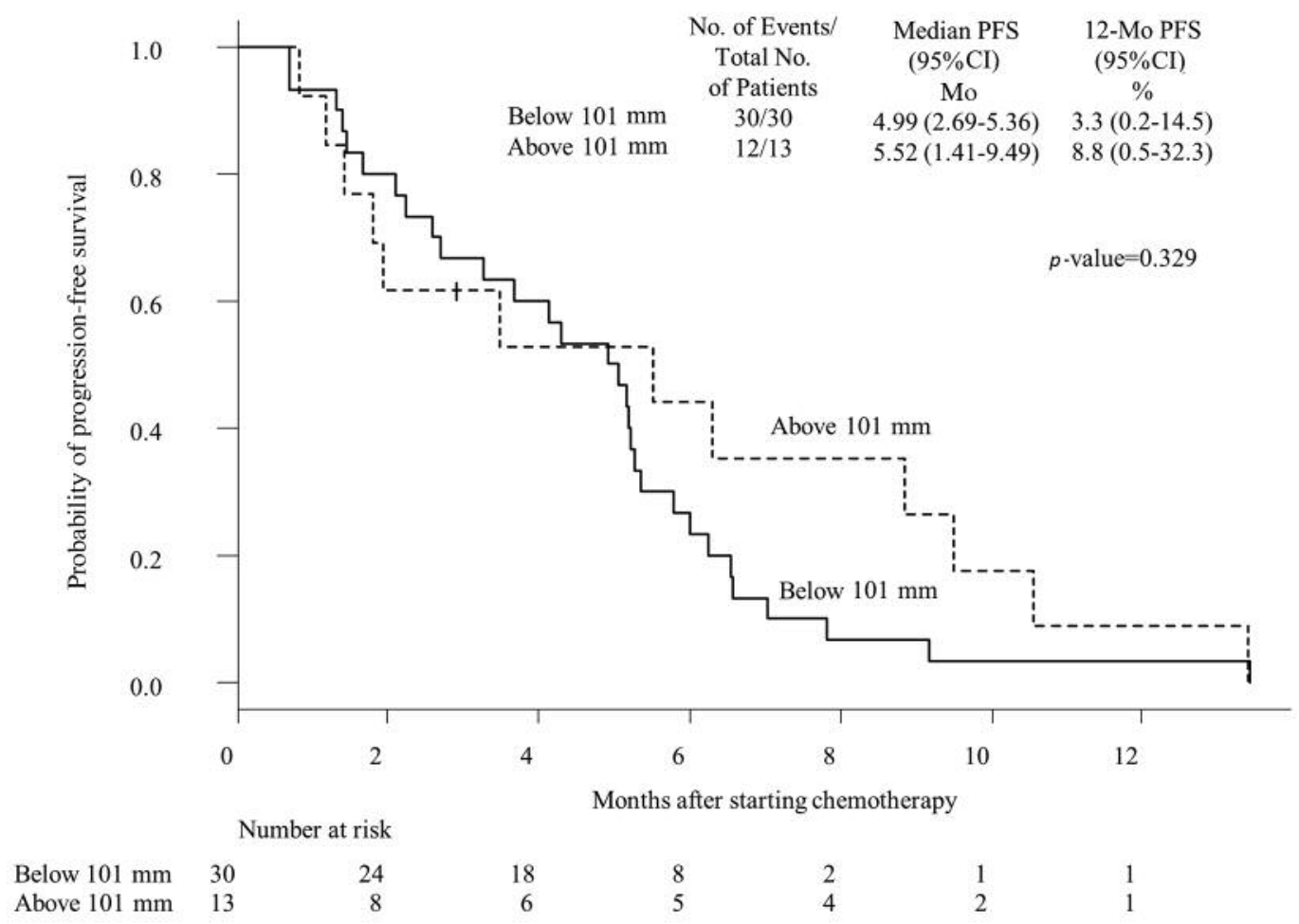

Figure 2. Kaplan-Meier (KM) plots grouped by patients with below and above median Baseline Tumor Size. KM plots for progression-free survival (PFS) after starting chemotherapy. Mo: Months.

3 Chen DS and Mellman I: Oncology meets immunology: The cancer-immunity cycle. Immunity 39(1): 1-10, 2013. PMID: 23890059, DOI: 10.1016/j.immuni.2013.07.012

4 Chen DS, Irving BA and Hodi FS: Molecular pathways: Nextgeneration immunotherapy - inhibiting programmed deathligand 1 and programmed death-1. Clin Cancer Res 18(24): 6580-6587, 2012. PMID: 23087408, DOI: 10.1158/10780432.CCR-12-1362

5 Wang C, Thudium KB, Han M, Wang XT, Huang H, Feingersh D, Garcia C, Wu Y, Kuhne M, Srinivasan M, Singh S, Wong S, Garner N, Leblanc H, Bunch RT, Blanset D, Selby MJ and Korman AJ: In vitro characterization of the anti-pd-1 antibody nivolumab, bms-936558, and in vivo toxicology in non-human primates. Cancer Immunol Res 2(9): 846-856, 2014. PMID: 24872026, DOI: 10.1158/2326-6066.CIR-14-0040

6 Brahmer J, Reckamp KL, Baas P, Crino L, Eberhardt WE, Poddubskaya E, Antonia S, Pluzanski A, Vokes EE, Holgado E, Waterhouse D, Ready N, Gainor J, Aren Frontera O, Havel L, Steins M, Garassino MC, Aerts JG, Domine M, Paz-Ares L, Reck M, Baudelet C, Harbison CT, Lestini B and Spigel DR: Nivolumab versus docetaxel in advanced squamous-cell nonsmall-cell lung cancer. N Engl J Med 373(2): 123-135, 2015. PMID: 26028407, DOI: 10.1056/NEJMoa1504627

7 Borghaei H, Paz-Ares L, Horn L, Spigel DR, Steins M, Ready NE, Chow LQ, Vokes EE, Felip E, Holgado E, Barlesi F, Kohlhaufl M, Arrieta O, Burgio MA, Fayette J, Lena H, Poddubskaya E, Gerber DE, Gettinger SN, Rudin CM, Rizvi N, Crino L, Blumenschein GR Jr., Antonia SJ, Dorange C, Harbison
CT, Graf Finckenstein F and Brahmer JR: Nivolumab versus docetaxel in advanced nonsquamous non-small-cell lung cancer. N Engl J Med 373(17): 1627-1639, 2015. PMID: 26412456, DOI: 10.1056/NEJMoa1507643

8 Herbst RS, Baas P, Kim DW, Felip E, Perez-Gracia JL, Han JY, Molina J, Kim JH, Arvis CD, Ahn MJ, Majem M, Fidler MJ, de Castro G, Jr., Garrido M, Lubiniecki GM, Shentu Y, Im E, Dolled-Filhart $\mathrm{M}$ and Garon $\mathrm{EB}$ : Pembrolizumab versus docetaxel for previously treated, pd-11-positive, advanced nonsmall-cell lung cancer (keynote-010): A randomised controlled trial. Lancet 387(10027): 1540-1550, 2016. PMID: 26712084, DOI: $10.1016 / \mathrm{S} 0140-6736(15) 01281-7$

9 Rittmeyer A, Barlesi F, Waterkamp D, Park K, Ciardiello F, von Pawel J, Gadgeel SM, Hida T, Kowalski DM, Dols MC, Cortinovis DL, Leach J, Polikoff J, Barrios C, Kabbinavar F, Frontera OA, De Marinis F, Turna H, Lee JS, Ballinger M, Kowanetz M, He P, Chen DS, Sandler A and Gandara DR: Atezolizumab versus docetaxel in patients with previously treated non-small-cell lung cancer (oak): A phase 3, open-label, multicentre randomised controlled trial. Lancet 389(10066): 255-265, 2017. PMID: 27979383, DOI: 10.1016/S0140-6736(16)32517-X

10 Antonia SJ, Villegas A, Daniel D, Vicente D, Murakami S, Hui R, Yokoi T, Chiappori A, Lee KH, de Wit M, Cho BC, Bourhaba M, Quantin X, Tokito T, Mekhail T, Planchard D, Kim YC, Karapetis CS, Hiret S, Ostoros G, Kubota K, Gray JE, Paz-Ares L, de Castro Carpeno J, Wadsworth C, Melillo G, Jiang H, Huang Y, Dennis PA and Ozguroglu M: Durvalumab after chemoradiotherapy in stage iii non-small-cell lung cancer. $\mathrm{N}$ 
Engl J Med 377(20): 1919-1929, 2017. PMID: 28885881, DOI: 10.1056/NEJMoa1709937

11 Carbone DP, Reck M, Paz-Ares L, Creelan B, Horn L, Steins M, Felip E, van den Heuvel MM, Ciuleanu TE, Badin F, Ready N, Hiltermann TJN, Nair S, Juergens R, Peters S, Minenza E, Wrangle JM, Rodriguez-Abreu D, Borghaei H, Blumenschein GR, Jr., Villaruz LC, Havel L, Krejci J, Corral Jaime J, Chang $\mathrm{H}$, Geese WJ, Bhagavatheeswaran P, Chen AC and Socinski MA: First-line nivolumab in stage iv or recurrent non-small-cell lung cancer. N Engl J Med 376(25): 2415-2426, 2017. PMID: 28636851, DOI: 10.1056/NEJMoa1613493

12 Funazo T, Nomizo T and Kim YH: Liver metastasis is associated with poor progression-free survival in patients with non-small cell lung cancer treated with nivolumab. J Thorac Oncol 12(9): e140e141, 2017. PMID: 28838713, DOI: 10.1016/j.jtho.2017.04.027

13 Gopalakrishnan V, Spencer CN, Nezi L, Reuben A, Andrews MC, Karpinets TV, Prieto PA, Vicente D, Hoffman K, Wei SC, Cogdill AP, Zhao L, Hudgens CW, Hutchinson DS, Manzo T, Petaccia de Macedo M, Cotechini T, Kumar T, Chen WS, Reddy SM, Szczepaniak Sloane R, Galloway-Pena J, Jiang H, Chen PL, Shpall EJ, Rezvani K, Alousi AM, Chemaly RF, Shelburne S, Vence LM, Okhuysen PC, Jensen VB, Swennes AG, McAllister F, Marcelo Riquelme Sanchez E, Zhang Y, Le Chatelier E, Zitvogel L, Pons N, Austin-Breneman JL, Haydu LE, Burton EM, Gardner JM, Sirmans E, Hu J, Lazar AJ, Tsujikawa T, Diab A, Tawbi H, Glitza IC, Hwu WJ, Patel SP, Woodman SE, Amaria RN, Davies MA, Gershenwald JE, Hwu P, Lee JE, Zhang J, Coussens LM, Cooper ZA, Futreal PA, Daniel CR, Ajami NJ, Petrosino JF, Tetzlaff MT, Sharma P, Allison JP, Jenq RR and Wargo JA: Gut microbiome modulates response to anti-pd-1 immunotherapy in melanoma patients. Science 359(6371): 97103, 2018. PMID: 29097493, DOI: 10.1126/science.aan4236

14 Kiriu T, Yamamoto M, Nagano T, Hazama D, Sekiya R, Katsurada M, Tamura D, Tachihara M, Kobayashi K and Nishimura Y: The time-series behavior of neutrophil-tolymphocyte ratio is useful as a predictive marker in non-small cell lung cancer. PLoS One 13(2): e0193018, 2018. PMID: 29447258, DOI: 10.1371/journal.pone.0193018

15 Joseph RW, Elassaiss-Schaap J, Kefford RF, Hwu WJ, Wolchok JD, Joshua AM, Ribas A, Hodi FS, Hamid O, Robert C, Daud AI, Dronca RS, Hersey P, Weber JS, Patnaik A, de Alwis DP, Perrone AM, Zhang J, Kang SP, Ebbinghaus SW, Anderson KM and Gangadhar T: Baseline tumor size is an independent prognostic factor for overall survival in patients with melanoma treated with pembrolizumab. Clin Cancer Res, 2018. PMID: 29685882, DOI: 10.1158/1078-0432.CCR-17-2386

16 Detterbeck FC, Boffa DJ, Kim AW and Tanoue LT: The eighth edition lung cancer stage classification. Chest 151(1): 193-203, 2017. PMID: 27780786, DOI: 10.1016/j.chest.2016.10.010

17 Budczies J, Klauschen F, Sinn BV, Gyorffy B, Schmitt WD, Darb-Esfahani S and Denkert C: Cutoff finder: A comprehensive and straightforward web application enabling rapid biomarker cutoff optimization. PLoS One 7(12): e51862, 2012. PMID: 23251644, DOI: 10.1371/journal.pone.0051862

18 Kanda Y: Investigation of the freely available easy-to-use software 'ezr' for medical statistics. Bone Marrow Transplant 48(3): 452458, 2013. PMID: 23208313, DOI: 10.1038/bmt.2012.244

19 Kobayashi H, Omori S, Nakashima K, Wakuda K, Ono A, Kenmotsu H, Naito T, Murakami H, Endo M and Takahashi T: Response to the treatment immediately before nivolumab monotherapy may predict clinical response to nivolumab in patients with non-small cell lung cancer. Int J Clin Oncol 22(4): 690-697, 2017. PMID: 28382561, DOI: 10.1007/s10147-017$1118-\mathrm{x}$

20 Raju B, Haug SR, Ibrahim SO and Heyeraas KJ: High interstitial fluid pressure in rat tongue cancer is related to increased lymph vessel area, tumor size, invasiveness and decreased body weight. J Oral Pathol Med 37(3): 137-144, 2008. PMID: 18251937 , DOI: $10.1111 / \mathrm{j} .1600-0714.2007 .00602 . \mathrm{x}$

21 Schaaf MB, Garg AD and Agostinis P: Defining the role of the tumor vasculature in antitumor immunity and immunotherapy. Cell Death Dis 9(2): 115, 2018. PMID: 29371595, DOI: 10.1038/s41419-017-0061-0

22 Arsham AM, Plas DR, Thompson CB and Simon MC: Akt and hypoxia-inducible factor-1 independently enhance tumor growth and angiogenesis. Cancer Res 64(10): 3500-3507, 2004. PMID: 15150104, DOI: 10.1158/0008-5472.CAN-03-2239

23 Fukumura D, Kloepper J, Amoozgar Z, Duda DG and Jain RK: Enhancing cancer immunotherapy using antiangiogenics: Opportunities and challenges. Nat Rev Clin Oncol 15(5): 325340, 2018. PMID: 29508855, DOI: 10.1038/nrclinonc.2018.29

24 Wang Q, Hu DF, Rui Y, Jiang AB, Liu ZL and Huang LN: Prognosis value of hif-1alpha expression in patients with nonsmall cell lung cancer. Gene 541(2): 69-74, 2014. PMID: 24631267, DOI: 10.1016/j.gene.2014.03.025

25 Nagarsheth N, Wicha MS and Zou W: Chemokines in the cancer microenvironment and their relevance in cancer immunotherapy. Nat Rev Immunol 17(9): 559-572, 2017. PMID: 28555670, DOI: 10.1038/nri.2017.49

26 Maisel K, Sasso MS, Potin L and Swartz MA: Exploiting lymphatic vessels for immunomodulation: Rationale, opportunities, and challenges. Adv Drug Deliv Rev 114: 43-59, 2017. PMID: 28694027, DOI: 10.1016/j.addr.2017.07.005

27 Binnewies M, Roberts EW, Kersten K, Chan V, Fearon DF, Merad M, Coussens LM, Gabrilovich DI, Ostrand-Rosenberg S, Hedrick CC, Vonderheide RH, Pittet MJ, Jain RK, Zou W, Howcroft TK, Woodhouse EC, Weinberg RA and Krummel MF: Understanding the tumor immune microenvironment (time) for effective therapy. Nat Med 24(5): 541-550, 2018. PMID: 29686425, DOI: 10.1038/s41591-018-0014-x

28 Huber V, Camisaschi C, Berzi A, Ferro S, Lugini L, Triulzi T, Tuccitto A, Tagliabue E, Castelli C and Rivoltini L: Cancer acidity: An ultimate frontier of tumor immune escape and a novel target of immunomodulation. Semin Cancer Biol 43: 74-89, 2017. PMID: 28267587, DOI: 10.1016/j.semcancer.2017.03.001

29 Huang AC, Postow MA, Orlowski RJ, Mick R, Bengsch B, Manne S, Xu W, Harmon S, Giles JR, Wenz B, Adamow M, Kuk D, Panageas KS, Carrera C, Wong P, Quagliarello F, Wubbenhorst B, D'Andrea K, Pauken KE, Herati RS, Staupe RP, Schenkel JM, McGettigan S, Kothari S, George SM, Vonderheide RH, Amaravadi RK, Karakousis GC, Schuchter LM, Xu X, Nathanson KL, Wolchok JD, Gangadhar TC and Wherry EJ: T-cell invigoration to tumour burden ratio associated with anti-pd-1 response. Nature 545(7652): 60-65, 2017. PMID: 28397821, DOI: 10.1038/nature22079

Received December 19, 2018

Revised January 5, 2019

Accepted January 7, 2019 